\title{
Die Bearbeiter der 27. Auflage
}

Jörg-Peter Becker, Vorsitzender Richter am Bundesgerichtshof a. D., Karlsruhe und Obernburg

Dr. Johannes Berg, Richter am Bundesgerichtshof, Karlsruhe

Dr. Camilla Bertheau, Rechtsanwältin in Berlin

Gabriele Cirener, Vorsitzende Richterin am Bundesgerichtshof, Leipzig

Dr. Volker Erb, Professor an der Johannes Gutenberg-Universität Mainz

Dr. Robert Esser, Professor an der Universität Passau

Dr. Karsten Gaede, Professor an der Bucerius Law School, Hamburg

Dr. Klaus Ferdinand Gärditz, Professor an der Rheinischen Friedrich-Wilhelms-Universität Bonn, Richter am OVG Nordrhein-Westfalen im Nebenamt, stellvertretender Richter am VerfGH Nordrhein-Westfalen

Kerstin Gärtner, Richterin am Kammergericht

Dr. Dirk Gittermann, Richter am Oberlandesgericht Celle

Dr. Sabine Gleß, Professorin an der Universität Basel

Dr. Dr. h.c. Karl Heinz Gössel, em. Professor an der Friedrich-Alexander-Universität Erlangen-Nürnberg, Richter am Bayerischen Obersten Landesgericht a. D., München

Dr. Kirsten Graalmann-Scheerer, Generalstaatsanwältin in Bremen, Honorarprofessorin an der Hochschule für öffentliche Verwaltung in Bremen

Klaus-Peter Hanschke, Richter am Kammergericht

Dr. Pierre Hauck, Professor an der Justus-Liebig-Universität Gießen

Dr. Hans Hilger, Ministerialdirektor im Bundesministerium der Justiz a. D., Bad Honnef

Dr. Dr. Alexander Ignor, Rechtsanwalt in Berlin, Apl. Professor an der Humboldt-Universität zu Berlin

Dr. Christian Jäger, Professor an der Friedrich-Alexander-Universität Erlangen-Nürnberg

Dr. Matthias Jahn, Professor an der Goethe-Universität Frankfurt am Main, Richter am Oberlandesgericht Frankfurt a. M.

Dr. Björn Jesse, Richter am Landgericht Berlin

Dr. Pascal Johann, Rechtsanwalt in Frankfurt a. M.

Dr. Daniel M. Krause, Rechtsanwalt in Berlin

Dr. Matthias Krauß, Bundesanwalt beim Bundesgerichtshof, Karlsruhe

Dr. Dr. h.c. mult. Hans-Heiner Kühne, em. Professor an der Universität Trier

Detlef Lind, Richter am Kammergericht

Dr. Holger Matt, Rechtsanwalt in Frankfurt am Main, Honorarprofessor an der Goethe-Universität Frankfurt am Main

Dr. Markus Mavany, Richter am Landgericht Trier

Dr. Eva Menges, Richterin am Bundesgerichtshof, Karlsruhe

Dr. Andreas Mosbacher, Richter am Bundesgerichtshof, Leipzig, Honorarprofessor an der Universität Leipzig

Dr. Ali B. Norouzi, Rechtsanwalt in Berlin

Dr. Günther M. Sander, Vorsitzender Richter am Bundesgerichtshof, Leipzig, Honorarprofessor an der Humboldt-Universität zu Berlin

Dr. Frank Peter Schuster, Professor an der Julius-Maximilians-Universität Würzburg

Dr. Wolfgang Siolek, Vorsitzender Richter am Oberlandesgericht Celle a. D.

Dr. Carl-Friedrich Stuckenberg, Professor an der Rheinischen Friedrich-Wilhelms-Universität Bonn

Dr. Michael Tsambikakis, Rechtsanwalt in Köln, Honorarprofessor an der Universität Passau

Dr. Brian Valerius, Professor an der Universität Bayreuth

Marc Wenske, Richter am Bundesgerichtshof, Karlsruhe

Dr. Raik Werner, Richter am Oberlandesgericht München 
